\title{
Repositioning of guanabenz in conjugation with gold and silver nanoparticles against pathogenic amoebae Acanthamoeba castellanii and Naegleria fowleri
}

\author{
Areeba Anwar ${ }^{\mathrm{a}}$, Mohammad Ridwane Mungroo $^{\mathrm{a}}$, Ayaz Anwar ${ }^{\mathrm{a}}$, William J. Sullivan Jr ${ }^{\mathrm{b}}$, \\ Naveed Ahmed Khan ${ }^{\mathrm{a}, \mathrm{c}}$, and Ruqaiyyah Siddiqui ${ }^{\mathrm{a}, \mathrm{c}}$ \\ ${ }^{a}$ Department of Biological Sciences, School of Science and Technology, Sunway University, \\ 5 Jalan Universiti, Bandar Sunway, Petaling Jaya, 47500, Selangor, Malaysia. \\ ${ }^{b}$ Department of Pharmacology and Toxicology, Indiana University School of Medicine, 635 \\ Barnhill Drive, Indianapolis, IN 46202, USA. \\ ${ }^{c}$ Department of Biology, Chemistry and Environmental Sciences, College of Arts and \\ Sciences, American University of Sharjah, Sharjah, 26666, United Arab Emirates
}

\section{Short title: Antiamoebic guanabenz coated metallic nanoparticles}

\section{*Corresponding author:}

\author{
Ayaz Anwar \\ Department of Biological Sciences, School of Science and Technology, Sunway University, \\ Subang Jaya, 47500, Selangor, Malaysia \\ Tel.: +603-56358630, Ext: 7119; Fax: +92-21-34819018 \\ Email address: ayazanwarkk@yahoo.com
}

This is the author's manuscript of the article published in final edited form as:

Anwar, A., Mungroo, M. R., Anwar, A., Sullivan, W., Khan, N. A., \& Siddiqui, R. (2019). Repositioning of guanabenz in conjugation with gold and silver nanoparticles against pathogenic amoebae Acanthamoeba castellanii and Naegleria fowleri. ACS Infectious Diseases. https://doi.org/10.1021/acsinfecdis.9b00263 
Brain-eating amoebae cause devastating infections of central nervous system in humans, having a mortality rate of $95 \%$. There are limited effective therapeutic options available clinically for treating granulomatous amoebic encephalitis and primary amoebic meningoencephalitis caused by Acanthamoeba castellanii and Naegleria fowleri, respectively. Here we report for the first time that guanabenz conjugated to gold and silver nanoparticles has significant antiamoebic activity against both A. castellanii and N. fowleri. Gold and silver conjugated guanabenz nanoparticles were synthesized by one-phase reduction method and were characterized by ultraviolet-visible spectrophotometry and atomic force microscopy. Both metals were facilely stabilized by the coating of guanabenz which was examined by surface plasmon resonance determination. The average size of gold nanoconjugated guanabenz was found to be $60 \mathrm{~nm}$, whereas, silver nanoparticles were produced in larger size distribution with the average diameter of around $100 \mathrm{~nm}$. Guanabenz and its noble metal nanoconjugates exhibited potent antiamoebic effects in the range of 2.5 to $100 \mu \mathrm{M}$ against both amoebae. Nanoparticles conjugation enhanced the antiamoebic effects of guanabenz, as more potent activity was observed at lower effective concentration (2.5 and $5 \mu \mathrm{M})$ compared to drug alone. Moreover, encystation and excystation assays revealed that guanabenz inhibits the interconversion between the trophozoite and cyst forms of $A$. castellanii. Cysticdal effects against $N$. fowleri were also observed. Notably, pre-treatment of A. castellanii with guanabenz and its nanoconjugates exhibited significant reduction in the host cell cytopathogenicity from $65 \%$ to $38 \%$ and $2 \%$ in case of gold and silver nanoconjugates, respectively. Moreover, the cytotoxic evaluation of guanabenz and nanoconjugates revealed negligible cytotoxicity against human cells. Guanabenz is already approved for hypertension and crosses the blood-brain barrier; the results of our current study suggest that guanabenz and its conjugated gold and silver nanoparticles can be repurposed as a potential drug for treating brain eating amoebic infections. 
Keywords: Guanabenz, Brain-eating amoeba, Acanthamoeba, Naegleria fowleri, Nanoparticles, Nanomedicine

Life threatening infectious diseases are caused by pathogenic free-living amoebae (FLA) such as Acanthamoeba species and Naegleria fowleri, which have devastating mortality rates. ${ }^{1}$ FLA are eukaryotic microorganism existing in two or three stages during their life cycle. Acanthamoeba species alternate between trophozoites and cysts, while additionally Naegleria fowleri is also found in a third stage called flagellate. Acanthamoeba castellanii causes life-threatening chronic central nervous system (CNS) disease granulomatous amoebic encephalitis (GAE), and the serious eye infection Acanthamoeba keratitis (AK). ${ }^{2} \mathrm{AK}$ is a localized, vision threatening, acute corneal infection that is most often encountered in contact lens wearers who maintain poor lens hygiene. ${ }^{3}$ GAE mostly occurs in immunocompromised patients and is characterized by cerebral edema, necrotic tissue areas, cortical and ganglial encephalomalacia. ${ }^{4}$ Following dissemination, Acanthamoeba cysts and trophozoites are detected in skin lesions, lungs, prostate and adrenal tissues other than CNS. ${ }^{5}$ On the other hand, acute and haemorrhagic CNS infection, primary amoebic meningoencephalitis (PAM) caused by Naegleria fowleri, has been reported in healthy individuals partaking in water activities, such as swimming, ablution, or diving. ${ }^{6}$ Naegleria fowleri gains access to the brain through the nasal route and causes extensive damage to the olfactory nerves and meninges. ${ }^{5}$ Necrotic neural haemorrhage is observed in all parts of the brain along with the detection of amoebic trophozoites, leading to poor prognosis of the patients and a $95 \%$ mortality rate. ${ }^{7,8}$

Currently, combination therapy is used to treat brain eating amoebic infections. The therapeutic options include different combinations of chlorhexidine, polyhexamethylene biguanides for $\mathrm{AK},{ }^{3}$ sulfadiazine, ketoconazole, fluconazole for GAE and amphotericin $\mathrm{B}$, 
rifampin, miconazole, and miltefosine for PAM.$^{9}$ However, these drugs have higher host cell cytotoxicity and poor efficacy towards the cyst forms. ${ }^{1,10}$ Thus, discovery of novel antiamoebic compounds having the ability to target both the trophozoite and cyst forms of FLA is required for the development of better therapeutic options.

Use of nanomedicine and repurposing of clinically approved drugs are useful strategies to treat brain eating infections. Nanoparticle-conjugated drug formulations have offered multiple benefits in the treatment of various diseases. ${ }^{11,12}$ Gold and silver nanoparticles have been studied extensively for their biological and medicinal properties. Nanoparticles (NP) are reported to improve drug carrier and delivery systems, bioavailability, and chemotherapeutic efficacy. Conjugation of compounds with NPs have exhibited antimicrobial, antiviral, antiangiogenic, wound healing and antiamoebic activities. ${ }^{13,14}$ Due to their size $(1-500 \mathrm{~nm})$, nanoparticles provide large surface to volume ratios and are permeable to cellular membranes for the interaction with biomolecules. ${ }^{15}$

We have previously reported the potential of guanabenz (GNB) as an antiparasitic agent against apicomplexan protozoan parasites Toxoplasma gondii and Plasmodium falciparum. ${ }^{16,17}$ Guanabenz acetate is FDA-approved for the treatment of hypertension. It is an $\alpha 2$ - adrenergic receptor agonist and has the ability to cross the blood-brain barrier. ${ }^{18}$ As an antiparasitic agent, GNB appears to interfere with translational control mechanisms in Toxoplasma and Plasmodium falciparum, and also reduces brain cyst burden in BALB/c mice with latent toxoplasmosis. ${ }^{16,17}$ Given these features of guanabenz, we sought to determine whether guanabenz has active against pathogenic A. castellanii and N. fowleri. Moreover, GNB conjugated gold and silver nanoparticles (AuNPs and AgNPs) were synthesized to determine the enhanced chemotherapeutic effects against $A$. castellanii and $N$. fowleri. Our findings represent the first demonstration of the antiamoebic activity of GNB and its nanoconjugates against brain eating amoebae. 


\section{Results and Discussion}

\section{Characterization of guanabenz coated gold and silver nanoparticles}

Guanabenz was used to stabilize gold as well as silver nanoparticles. After successful formation of colloidal gold and silver suspensions with guanabenz, these were characterized by ultraviolet-visible spectroscopy and atomic force microscopy. The UV-vis spectra of metallic nanoconjugates of guanabenz presented in Fig. 1a shows the presence of characteristic surface plasmon resonance band at 380 and $540 \mathrm{~nm}$ corresponding to AgNPs and AuNPs respectively. The spectra were recorded after subsequent washing of nanoparticles obtained by centrifuging at $10000 \mathrm{x} \mathrm{g}$ and resuspending in ultrapure deionized water. Atomic force microscopy imaging was carried out to determine the morphology of these synthesized nanoconjugates. The representative images of GNB-AuNPs and GNBAgNPs are presented in Fig. $1 \mathrm{~b}$ and 1c, respectively. The results show that gold nanoparticles were found to be smaller in size and relatively monodispersed as compared to AgNPs, which were a mixture of small particles and large aggregates. The size of GNB-AuNPs and GNBAgNPs was found to be in the broad range of 50-70 and 50-150 nm, respectively.

\section{Gold and silver conjugated guanabenz nanoparticles exhibited antiamoebic effects against Acanthamoeba castellanii}

Antiamoebic assays were carried out to determine the effects of GNB alone and GNB conjugated nanoparticles against $A$. castellanii (Fig. 2). Chlorhexidine was used as the positive control. GNB alone was tested at the concentrations of $2.5,5,10,25,50$, and 100 $\mu \mathrm{M}$. The concentrations used for the GNB conjugated with AuNPs and AgNPs were 2.5 and $5 \mu \mathrm{M}$. The statistical significance of the effects of drug conjugated nanoparticles on Acanthamoeba was evaluated against GNB alone. The results in Figure 2 show that GNB and 
its conjugated nanoparticles have significant antiamoebic effects against Acanthamoeba. However, conjugation of GNB with gold and silver nanoparticles exhibited enhanced and significant $(* P<0.05)$ antiamoebic effect as compared to the drug alone. GNB-AgNPs at 5 $\mu \mathrm{M}$ reduced the viability from $5.9 \times 10^{5}$ to $9.3 \times 10^{4}$ while in case of GNB alone the number of amoebae was found to be $3.8 \times 10^{5}$. Furthermore, treatment with bare gold and silver nanoparticles did not have any adverse effects as compared to untreated amoebae.

\section{Gold and silver conjugated guanabenz nanoparticles inhibited encystation of Acanthamoeba castellanii}

A. castellanii trophozoites were incubated in encystation medium with GNB alone or as metal-conjugated nanoparticles to determine their effects on encystment of $A$. castellanii. The encystation media induced the formation of $A$. castellanii cysts in negative controls. The treatment of amoebic trophozoites with GNB conjugated nanoparticles significantly inhibited $(* P<0.05$, two-sample $\mathrm{t}$ test and two-tailed distribution) the process of encystation, in comparison to the amoebae treated with GNB and unconjugated nanoparticles alone (Fig. 3).

\section{Gold and silver conjugated guanabenz nanoparticles inhibited excystation of}

\section{Acanthamoeba castellanii}

The potential of GNB alone and its conjugated nanoparticles were also evaluated against the cysts of Acanthamoeba castellanii. The amoebic cysts were dispersed in growth medium PYG, which were transformed into healthy trophozoites and enumerated using a haemocytometer. The treatment of amoebae with GNB conjugated gold and silver nanoparticles significantly inhibited the transformation of cysts into healthy trophozoites $(* P$ $<0.05$ ) at $5 \mu \mathrm{M}$ concentration, as compared to GNB and bare nanoparticles alone (Fig. 4). 
However, $2.5 \mu \mathrm{M}$ concentration of both gold and silver conjugated GNB nanoparticles did not show any inhibition in comparison with the respective controls.

\section{Gold and silver conjugated guanabenz nanoparticles reduced Acanthamoeba mediated host cell cytopathogenicity in $\mathrm{HaCaT}$ cells}

Cytopathogenicity assays were performed to test the effect of guanabenz conjugated nanoparticles on $A$. castellanii infected host cells (Fig. 5). The results revealed that, without any drug treatment, A. catellanii destroyed $65 \%$ of the host cells. Upon pre-treatment with chlorhexidine, the cytopathogenicity was completely diminished. GNB-AgNPs also significantly reduced the host cell death to $7.5 \%$ and $2.5 \%$, respectively at 2.5 and $5 \mu \mathrm{M}$. Guanabenz alone did not reduce cytopathogenicity, nor did the GNB-AuNPs when compared with AuNPs alone (Fig. 5). These findings are in accordance with the results of the antiamoebic assays (Fig. 2).

\section{Gold and silver conjugated guanabenz nanoparticles exhibited antiamoebic effects against Naegleria fowleri}

The antiamoebic activity of GNB and its nanoconjugates was also evaluated against N. fowleri (Fig. 6). Amphotericin B was used as the positive control. The results show a significant ( $* \mathrm{P}<0.05$, two-sample t test and two-tailed distribution) enhancement in antiamoebic activity of GNB conjugated nanoconjugates as compared to GNB alone. GNB alone only showed antiamoebic effects at 50 and $100 \mu \mathrm{M}$ against $N$. fowleri. However, GNBAuNPs and GNB-AgNPs produced potent antiamoebic effects at concentrations as low as 2.5 $\mu \mathrm{M}$. Conjugation of gold and silver exhibited equally effective antiamoebic effects as compared to gold and silver alone, which did not show any effects on amoebae. 


\section{Gold and Silver conjugated Guanabenz Nanoparticles exhibited Anticystic Effects against Naegleria fowleri}

The anticystic activities of GNB, GNB-AuNPs and GNB-AgNPs were also evaluated against cyst form of N. fowleri (Fig. 7). Amphotericin B was used as the positive control, whereas gold and silver alone were used as the negative controls. The results showed that 100 $\mu \mathrm{M}$ concentration of GNB alone induced significant $(* \mathrm{P}<0.05$, two-sample $\mathrm{t}$ test and twotailed distribution) anticystic effects as compared to the amoebae alone. However, the same effect was observed by the GNB nanoconjugates at $5 \mu \mathrm{M}$ concentration (\#P $<0.05$, twosample t test and two-tailed distribution), exhibiting the greater anticystic activity of GNB conjugated nanoconjugates in comparison to GNB alone.

\section{Gold and silver conjugated guanabenz nanoparticles did not exhibit cell cytotoxicity against HeLa and HaCaT cells}

The in vitro cell cytotoxicity was evaluated against two human cell lines by using Lactate dehydrogenase assay and the results are presented in Fig. 8 (a) HeLa cells and (b) human keratinocytes HaCaT cells. Consistent with GNB being a safe, FDA-approved drug, no significant toxicity was observed on these cell lines; only at $100 \mu \mathrm{M}$ did GNB cause $\sim 30 \%$ toxicity against $\mathrm{HaCaT}$ cells. For the GNB nanoconjugates, virtually no toxicity was observed for gold, and only very modest levels of toxicity were observed for silver, all $<20 \%$ in both human cell lines. The silver nanoconjugates of GNB also offered a little protection against the nanoparticles alone for HeLa cells. In all cases, the toxicity of GNB conjugated nanoparticles never exceeded the degree observed for the nanoparticles alone.

Different classes of drugs have been used as a curative treatment for brain-eating amoebic infections, but they remain ineffective. ${ }^{3,19,20} \mathrm{CNS}$ infections caused by 
Acanthamoeba and N. fowleri are fatal in almost all cases. The mortality rate exceeding $90 \%$ underscores the seriousness of lacking effective therapeutic options. Thus, there is an urgent need to identify new molecules for treating infections caused by the brain-eating amoebae. Drugs used clinically for treating Acanthamoeba infections tend to be resistant towards the dormant cyst form. ${ }^{21}$ Moreover, adverse effects such as host cell cytotoxicity, and inadequate drug delivery to the target site, remain major challenges. The synergistic therapy used for the treatment of brain eating amoebic infections includes different combinations of chlorhexidine, polyhexamethylene biguanides for AK, pentamidines, azoles, sulfadiazine for GAE and amphotericin B, rifampin, azoles, and miltefosine for PAM.

Recently, various classes of natural and synthetic compounds and materials have shown potential antiamoebic activity in-vitro and in-vivo. Repurposing of clinically approved drugs for other diseases have been of great interest for the treatment against brain eating amoebae induced infections. Guanabenz acetate is an FDA-approved drug and is used to treat hypertension. It is an agonist for $\alpha-2$ adrenergic receptor and the maximum dose studied is 32 mg twice a day for patients above 18 years of age. ${ }^{18}$ Although GNB is well tolerated with few side effects, it can cause adverse toxicity among patients with liver impairment. In previous studies, we reported the antiparasitic potential of GNB against Toxoplasma, which included efficacy against both proliferating (tachyzoite) and encysted (bradyzoite) forms. ${ }^{16}$ Importantly, GNB also reduced brain cyst burdens in mice chronically infected with Toxoplasma. This might be due, in part, to the ability of guanabenz to cross the blood-brain barrier. ${ }^{16}$ These features prompted our testing of GNB against brain eating amoebae, i.e., Acanthamoeba and N. fowleri. Furthermore, we also synthesized nanoconjugates of GNB with gold and silver to evaluate the effects against both trophozoites and cyst forms. Nanoparticle-conjugated drugs have shown great potential in biomedicine in terms of increased efficacy, bioavailability, targeted drug delivery, and theranostics. ${ }^{22}$ We have 
previously reported the potential of nanoparticles to enhance antiamoebic activity after the conjugation with several compounds, such as chlorhexidine, amphotericin B, cinnamic acid, diazepam, phenytoin etc. (Aqeel et al., 2016, Anwar et al., 2018, Anwar et al., 2019b). ${ }^{23-25}$ Due to the small size in nanometers, increased solubility and stability, and reduced host cell cytotoxicity, nanoparticles have great potential and applications in biomedicine. The most frequently used inorganic nanoparticles are gold, silver, iron, cobalt, and titanium oxide. ${ }^{11}$ Gold has inert properties and less cytotoxicity, which enables it to be considered as nanomaterial of choice for therapeutics. Silver nanoparticles themselves have shown antimicrobial activity against a broad spectrum of microbes. ${ }^{26}$

In addition to its agonistic activity on $\alpha-2$ adrenergic receptors, GNB blocks the dephosphorylation of eIF $2 \alpha$, thereby disrupting translational control in cells. ${ }^{27}$ This ability may explain its activity against neurodegenerative diseases, ${ }^{28}$ toxoplasmosis ${ }^{29}$ and breast cancer. ${ }^{30}$ Disrupting translational control can be an effective strategy to target various stages of the life cycle of different pathogens. ${ }^{31}$ In the current study, antiamoebic assays demonstrated the amoebistatic potential of GNB alone, as well as the enhanced activity after the conjugation with gold and silver nanoparticles.

The transformation of trophozoites into cyst forms (encystation) and vice versa (excystation) involves metabolic changes inside the cellular and metabolic machinery of Acanthamoeba. ${ }^{2}$ In Acanthamoeba, propranolol, a $\beta$ adrenergic receptors antagonist showed reduction in cell viability and encystation along with declined protease activity. ${ }^{32}$ The reduced enumeration of cysts formed during encystation assay after the pre-treatment of amoebae with GNB and its nanoconjugates might be due to the action of GNB on adrenergic receptors. However, its agonistic or antagonistic activity against the multiple adrenergic receptor types has yet to be explored. Upregulated protein methylation has also been reported during encystation. The use of inhibitors of cellulase synthase, autophagy and cyst specific 
cysteine protease have also resulted in inhibition of encystation process. Phosphoglycerate dehydrogenase (PGDH) and phosphoserine aminotransferase (PSAT), key mediators of Lserine biosynthesis pathway are known to have important functions in amoebae. ${ }^{33} \mathrm{~L}$-serine is an important intermediate molecule of different metabolic pathways including synthesis of sphingomyelins, phospholipids and cerebrosides. ${ }^{34,35}$ Similar pattern of results was observed by the excystation assay proposing the effect of GNB on the metabolism of amoebic cells. These studies suggest the possible reason of inhibition of encystation and excystation of Acanthamoeba by the action of GNB alone and its nanoconjugates.

Deng et al., 2015 reported in their study that artemether, an antimalarial agent induced apoptosis (PCD) in Acanthamoeba. Furthermore, the proteome analysis revealed that artemisinin downregulates PGDH and PSAT. ${ }^{35}$ This further validates our rationale of repurposing of clinically approved drug for finding the acute and cost-effective methods of cure for treating brain eating amoebic infections. We have previously reported the potential use of CNS drugs such as diazepam and phenytoin against Acanthamoeba and N. fowleri. ${ }^{23}$ The evaluation of antiamoebic properties of GNB alone, GNB-AuNPs and GNB-AgNPs against $N$. fowleri also showed the decrease in number of viable amoebae, when compared to the untreated amoebic cells. Furthermore, the anticystic activity against $N$. fowleri was evaluated for the first time. GNB and its nanoconjugates were found to be also active against cyst form of $N$. fowleri. As, GNB is FDA approved drug, therefore it showed minor cytotoxicity against $\mathrm{HeLa}$ and $\mathrm{HaCaT}$ cell lines, indicating its broader therapeutic window in humans. ${ }^{17}$

GNB has also shown to have antiinflammatory activity in-vitro. The increased production of interleukin-10 (IL-10) and decreased levels of tumour necrotic factor alpha (TNF- $\alpha$ ) are induced by GNB in lipopolysaccharide (LPS) stimulated lethal model in mice, also preventing liver functional impairment and prolonging the mice survival. ${ }^{36}$ Inhibition of 
GADD34, an $\alpha 2$-adrenergic receptor agonist, by GNB treatment has shown the improved survival rate in human cells, when ER stressors were given. ${ }^{37,38}$ This suggests the possible mechanism of their ability to reduce cell cytopathogenicity. Pre-treatment of Acanthamoeba with drug and its conjugated nanoparticles also reduced the host cell pathogenicity in $\mathrm{HaCaT}$ cells. The current study also reveals the efficacy of GNB conjugated nanoparticles for the treatment of brain eating amoebic infections.

\section{Methods}

\section{Synthesis of guanabenz conjugated nanoparticles}

Guanabenz solution was made in phosphate buffer saline (PBS) and stored at $4{ }^{\circ} \mathrm{C}$. The nanoparticles were synthesized as described previously. ${ }^{39}$ The Au-conjugated guanabenz nanoparticles were synthesized by mixing $0.1 \mathrm{mM}$ of guanabenz solution and $0.1 \mathrm{mM}$ of Potassium gold chloride solution, in the ratios of $1: 1,1: 5$, and 5:1, and kept on magnetic stirring for 15 minutes. The reduction was achieved by adding $50 \mu \mathrm{L}$ of freshly prepared 4 mM sodium borohydride solution. The conjugation of GNB with AuNPs was indicated by change of transparent solution into pink coloured solution. The reaction mixture was kept on stirring for 1 hour to attain the maximum yield and stability. Similar procedure was followed for the synthesis of Ag-conjugated guanabenz nanoparticles, but the stock concentration used for guanabenz and silver nitrate solutions was $1 \mathrm{mM}$. Formation of GNB-AgNPs was indicated by the appearance of yellowish-brown colour in the reaction mixture. Bare nanoparticles (AuNPs and AgNPs alone) were also synthesized by similar method except for the addition of any drug or stabilizing agent. Guanabenz nanoconjugates were subjected to characterization by UV-Visible spectrophotometric determination of surface plasmon resonance bands using Thermo (Evolution 210) spectrophotometer, and morphological analysis was carried out on AFM using Agilent (AFM 5500) instrument. 


\section{Cell culture}

HeLa cells (cervical cancer cell line, ATCC-CCL-2) and HaCaT cells (human keratinocyte cell line) were cultured routinely, in RPMI-1640 medium supplemented with FBS (10\%), L-glutamine (1\%), nonessential amino acids (1\%), and antibiotics (1\% penicillin-streptomycin). The cells were maintained in monolayer form incubated at $37{ }^{\circ} \mathrm{C}$ in a $5 \% \mathrm{CO}_{2}$ humidified incubator. Sub culturing was done as per the requirement, according to the previously described protocol. ${ }^{40}$

\section{Acanthamoeba culture cultivation}

A. castellanii strain of genotype T4 was purchased from American type tissue collection (ATCC \# 50492). Acanthamoeba were cultured routinely in PYG medium in 75 $\mathrm{cm}^{2}$ tissue culture flasks and incubated at $30{ }^{\circ} \mathrm{C}$ under sterile conditions. The amoebae cultures in the flasks were replenished with fresh medium every alternate day. Before every experiment, medium was removed. The cells were washed with $10 \mathrm{ml} \mathrm{PBS}$, and $10 \mathrm{ml}$ fresh PYG medium was added. Then, adhered Acanthamoeba cells were detached by placing the flasks on ice for 15 minutes, followed by the gentle tapping. Cells were collected after centrifugation (3000 g / $10 \mathrm{~min})$ and seeded for the subsequent experiment. This procedure was followed in all the assays. ${ }^{41}$

\section{Naegleria Culture Cultivation}

$N$. fowleri were grown in RPMI-1640 medium on the monolayers of HeLa cells as their food source, in $75 \mathrm{~cm}^{2}$ tissue culture flasks under sterile conditions $\left(37^{\circ} \mathrm{C}\right.$ and $5 \%$ $\mathrm{CO}_{2}$ ), as reported previously. The clinical isolate of $N$. fowleri used was isolated from the cerebrospinal fluid of the patient and purchased from American type tissue collection (ATCC \# 30174)..$^{40}$ 


\section{Antiamoebic Assay}

Briefly, amoebic trophozoites were seeded in 24 well plate in $\left(5 \times 10^{5} /\right.$ well / $500 \mu \mathrm{L}$ RPMI). Cells were incubated with $2.5 \mu \mathrm{M}, 5 \mu \mathrm{M}, 10 \mu \mathrm{M}, 25 \mu \mathrm{M}, 50 \mu \mathrm{M}$, and $100 \mu \mathrm{M}$ concentrations of GNB alone. The concentrations used for GNB-AuNPs and GNB-AgNPs were $2.5 \mu \mathrm{M}$ and $5 \mu \mathrm{M}$. Chlorhexidine $(100 \mu \mathrm{M})$ and Amphotericin $\mathrm{B}(100 \mu \mathrm{M})$ were added in Acanthamoeba and N. fowleri respectively, as the positive controls. Amoebae alone and solvent used were the negative controls. After 24 hours of incubation at $30{ }^{\circ} \mathrm{C}$ with the compounds, the trophozoites were enumerated by trypan blue $(0.1 \%)$ exclusion method, using a haemocytometer, as reported previously. ${ }^{42}$

\section{Encystation assay}

Acanthamoeba trophozoites were seeded in 24 well plate in $\left(5 \times 10^{5} /\right.$ well / $500 \mu \mathrm{L} /$ PBS), with the GNB alone, GNB-AuNPs and GNB AgNPs. As encystation medium (EM), each well was supplemented with $50 \mathrm{mM}$ magnesium chloride and $10 \%$ glucose. The cells were incubated for 72 hours at $30^{\circ} \mathrm{C}$ until the observation of cysts formation in the negative control (amoebae only with EM), under inverted microscope. Following the incubation, $0.1 \%$ SDS (sodium dodecyl sulphate) was added in each well to kill the remaining trophozoites. The SDS-resistant cysts were counted under an inverted microscope, using a hemocytometer. ${ }^{42}$

\section{Excystation assay}

Acanthamoeba castellanii trophozoites were transformed into cysts by their harvestation on non-nutrient agar plates. The plates were incubated at $30{ }^{\circ} \mathrm{C}$ for two weeks. Before experiment, the cysts were scraped carefully in PBS with the help of a cell scraper, followed by the centrifugation at $5000 \mathrm{~g}$ for 10 minutes. The collected cyst pellet was dissolved in PYG and number of cysts were counted. For the assay, cysts $\left(1 \times 10^{5} /\right.$ well $/ 500$ 
$\mu \mathrm{L}$ PYG) were incubated with the test compounds and incubated at $30{ }^{\circ} \mathrm{C}$ for 72 hours.

Following the incubation, the cysts converted into trophozoites were counted by trypan blue exclusion method, using hemocytometer. ${ }^{43}$

\section{Anticystic assay for $N$. fowleri}

N. fowleri cells were grown on HeLa monolayers as feeder cells. After the complete consumption of monolayers, the flasks were tapped gently to detach the amoebae and were collected by centrifugation at $1260 \mathrm{x}$ g. The cell pellet obtained was resuspended in RPMI1640 and transferred into a new $75 \mathrm{~cm}^{2}$ tissue culture flask. The cells were monitored closely for two weeks until transformed into cysts form. The cysts were collected by gentle tapping of the flasks, followed by the centrifugation. For the experiment, cysts $\left(1 \times 10^{5 /}\right.$ well $/ 500 \mu \mathrm{L}$ RPMI) were incubated with GNB and its nanoconjugates at $37^{\circ} \mathrm{C}$ for 72 hours. After incubation time, the remaining viable cysts were counted by trypan blue exclusion method, using hemocytometer.

\section{Acanthamoeba mediated host cell cytopathogenicity assay}

Amoebic cells ( $1 \times 10^{5} /$ well / $500 \mu \mathrm{L}$ RPMI) were incubated with the GNB alone and drug conjugated nanoparticles in 24 well plates for 2 hours at $30^{\circ} \mathrm{C}$. After incubation, the plates were kept on ice for 20 minutes to detach the adherent cells. All the test samples were collected in centrifuge tubes and centrifuged at $5000 \mathrm{~g}$ for 10 minutes. The collected cell pellets were washed twice with PBS. After washing, $200 \mu \mathrm{L}$ fresh RPMI were added to the obtained cell pellets. These test samples and their respective controls were added to the cultured monolayer of HeLa cells in 96 well plates, after old medium was removed. ${ }^{43}$ To determine the cytotoxicity by lactate dehydrogenase (LDH) assay, the HeLa cells with the added amoebic cells were incubated for 24 hours in the incubator at $37^{\circ} \mathrm{C}$ and $5 \% \mathrm{CO}_{2}$. After 24 hours, supernatants (cell-free) were collected and released LDH was measured by using 
LDH cytotoxicity assay detection kit as per the protocol, using the following formula: \% cytotoxicity $=($ absorbance of sample treated well - absorbance of untreated cells $) /$ (absorbance of cells treated with Triton X-100 - absorbance of untreated cells) $\times 100$.

\section{Host cell cytotoxicity assay}

The cytotoxicity induced by GNB and its conjugated metal nanoparticles was measured on two different cell lines. HaCaT and HeLa cells were seeded at a density of more than $50 \%$ confluency and incubated till the monolayer formation. After the monolayer formed, old medium was replaced with fresh RPMI supplemented with the designated concentrations of the test compounds and incubated for 24 hours. Following incubation, LDH was measured as per the protocol described previously. ${ }^{44}$

\section{Statistical analysis}

The data are represented as mean \pm standard error, and $n=\geq 3$. All the experiments were performed in duplicate and statistical analysis was performed. Two sample student $t$ test was applied to determine the significant difference among the groups with 2 paired distribution. The different numeric indicate a statistical difference for at least at $P<0.05$, while $* P<0.05, * * P<0.01$, and $* * * P<0.001$ with another group.

\section{Conclusion}

In conclusion, the current study demonstrates the antiamoebic activity of guanabenz against brain eating amoebae $A$. castellanii and $N$. fowleri for the first time. Furthermore, the conjugation of the drug with gold and silver nanoparticles enhanced the antiamoebic activity, suggesting its potential use in nanomedicine for efficient drug delivery and reduced adverse effects. However, detailed mechanistic studies are required to elucidate the molecular mechanism of action of guanabenz and its gold and silver nanoconjugates against brain eating amoebae. 


\section{Acknowledgements}

All authors agree to submit the current version. The work was supported by Sunway University Malaysia's Internal Grant (INT-SST-DBS-2019-02). Research in Dr. Sullivan's lab is supported by a grant for the National Institutes of Health (AI124723).

\section{Competing interest}

Authors declare no conflict of interest neither financial nor ethical.

\section{References}

1. Visvesvara, G.S., Moura, H., Schuster, F.L. (2007). Pathogenic and opportunistic free-living amoebae: Acanthamoeba spp., Balamuthia mandrillaris, Naegleria fowleri, and Sappinia diploidea. FEMS Immunol Med Microbiol 50, 1-26.

2. Khan, N.A. (2006). Acanthamoeba: biology and increasing importance in human health. FEMS Microbiol Rev 30, 564-595.

3. Lorenzo-Morales, J., Khan, N. A., Walochnik, J. (2015). An update on Acanthamoeba keratitis: diagnosis, pathogenesis and treatment. Parasite, 22.

4. Martinez, A.J. (1985). Free-Living Amoebas: Natural History, Prevention, Diagnosis, Pathology, and Treatment of Disease. CRC Press, Boca Raton, FL.

5. Ong, T. Y. Y., Khan, N. A., Siddiqui, R. (2017). Brain-eating amoebae: predilection sites in the brain and disease outcome. J Clin Microbiol 55, 1989-1997.

6. Yoder, J.S., Eddy, B.A., Visvesvara, G.S., Capewell, L., Beach, M.J. (2010). The epidemiology of primary amoebic meningoencephalitis in the USA, 1962-2008. Epidemiol Infect 138, 968-975.

7. Martinez, A.J., Visvesvara, G.S. (1997). Free-living, amphizoic and opportunistic amebas. Brain Pathol 7, 583-598. 
8. Schuster, F. L., Visvesvara, G. S. (2004). Opportunistic amoebae: challenges in prophylaxis and treatment. Drug Resist Updates 7, 41-51.

9. Mungroo, M.R., Anwar, A., Khan, N.A., Siddiqui, R. (2019). Brain-eating amoebae infection: challenges and opportunities in chemotherapy. Mini Rev Med Chem (e-pub ahead of print) doi:10.2174/1389557519666190313161854.

10. Lorenzo-Morales, J., Martín-Navarro, C. M., López-Arencibia, A., Arnalich-Montiel, F., Piñero, J. E., Valladares, B. (2013). Acanthamoeba keratitis: an emerging disease gathering importance worldwide?. Trends Parasitol 29, 181-187.

11. Patra, J. K., Das, G., Fraceto, L. F., Campos, E. V. R., del Pilar Rodriguez-Torres, M., Acosta-Torres, L. S., Diaz-Torres, L. A., Grillo, R., Swamy, M. K., Sharma, S., Habtemariam, S., Shin, H-S. (2018). Nano based drug delivery systems: recent developments and future prospects. J Nanobiotechnol 16, 71.

12. Gurunathan, S., Kang, M. H., Qasim, M., Kim, J. H. (2018). Nanoparticle-mediated combination therapy: Two-in-one approach for cancer. Int J Mol Sci 19, 3264.

13. Rai, M., Yadav, A., Gade, A. (2009). Silver nanoparticles as a new generation of antimicrobials. Biotechnol Adv 27, 76-83.

14. Anwar, A., Masri, A., Rao, K., Rajendran, K., Khan, N. A., Shah, M. R., Siddiqui, R. (2019a). Antimicrobial activities of green synthesized gums-stabilized nanoparticles loaded with flavonoids. Sci Rep 9, 3122.

15. Zazo, H., Colino, C.I., Lanao, J.M. (2016). Current applications of nanoparticles in infectious diseases. J Control Release 224, 86-102.

16. Benmerzouga, I., Checkley, L. A., Ferdig, M. T., Arrizabalaga, G., Wek, R. C., Sullivan, W. J. (2015). Guanabenz repurposed as an antiparasitic with activity against acute and latent toxoplasmosis. Antimicrob Agents Chemother 59, 6939-6945. 
17. Martynowicz, J., Augusto, L., Wek, R. C., Boehm, S. L., \& Sullivan, W. J. (2019). Guanabenz Reverses a Key Behavioral Change Caused by Latent Toxoplasmosis in Mice by Reducing Neuroinflammation. mBio 10, e00381-19.

18. Baum, T., Shropshire, A.T. (1976). Studies on the centrally mediated hypotensive activity of guanabenz. Eur J Pharmacol 37, 31-44.

19. Siddiqui, R., Khan, N.A. (2012). Biology and pathogenesis of Acanthamoeba. Parasit Vectors 5, 6 .

20. Martín-Navarro, C. M., López-Arencibia, A., Arnalich-Montiel, F., Valladares, B., Piñero, J. E., Lorenzo-Morales, J. (2013). Evaluation of the in vitro activity of commercially available moxifloxacin and voriconazole eye-drops against clinical strains of Acanthamoeba. Graefe's Arch Clin Exp Ophthalmol 251, 2111-2117.

21. Siddiqui, R., Aqeel, Y., Khan, N. A. (2016). The development of drugs against Acanthamoeba infections. Antimicrob Agents Chemother 60, 6441-6450.

22. Janib, S. M., Moses, A. S., MacKay, J. A. (2010). Imaging and drug delivery using theranostic nanoparticles. Adv Drug Delivery Rev 62, 1052-1063.

23. Anwar, A., Rajendran, K., Siddiqui, R., Raza Shah, M., Khan, N. A. (2018). Clinically approved drugs against CNS diseases as potential therapeutic agents to target brain-eating amoebae. ACS Chem Neurosci 10, 658-666.

24. Anwar, A., Siddiqui, R., Shah, M. R., Khan, N. A. (2019b). Gold Nanoparticles Conjugation Enhances Antiacanthamoebic Properties of Nystatin, Fluconazole and Amphotericin B. J Microbiol Biotechnol 29, 171-177.

25. Aqeel, Y., Siddiqui, R., Anwar, A., Shah, M. R., Khan, N. A. (2016). Gold nanoparticle conjugation enhances the antiacanthamoebic effects of chlorhexidine. Antimicrob Agents Chemother 60, 1283-1288. 
26. Sondi, I., Salopek-Sondi, B. (2004). Silver nanoparticles as antimicrobial agent: a case study on E. coli as a model for Gram-negative bacteria. J Colloid Interface Sci $275,177-182$.

27. Kang, H. J., Seol, H. S., Lee, S. E., Suh, Y. A., Kim, J., Jang, S. J., Yu, E. (2019). Guanabenz Acetate Induces Endoplasmic Reticulum Stress-Related Cell Death in Hepatocellular Carcinoma Cells. J Pathol Trans Med 53, 94-103.

28. Wang, L., Popko, B., Tixier, E., Roos, R.P. (2014). Guanabenz, which enhances the unfolded protein response, ameliorates mutant SOD1-induced amyotrophic lateral sclerosis. Neurobiol Dis 71, 317-24.

29. Vieira, F.G., Ping, Q., Moreno, A.J., Kidd, J. D., Thompson, K., Jiang, B., Lincecum, J. M., Wang, M. Z., De Zutter, G. S., Tassinari, V. R., Levine, B., Hatzipetros, T., Gill, A., Perrin, S. (2015). Guanabenz treatment accelerates disease in a mutant SOD1 mouse model of ALS. PLoS One 10, e0135570.

30. Hamamura, K., Minami, K., Tanjung, N., Wan, Q., Koizumi, M., Matsuura, N., Na, S., Yokota, H. (2014). Attenuation of malignant phenotypes of breast cancer cells through eIF2alpha-mediated downregulation of Rac1 signaling. Int J Oncol 44, 1980 8.

31. Holmes, M. J., da Silva Augusto, L., Zhang, M., Wek, R. C., \& Sullivan Jr, W. J. (2017). Translational control in the latency of apicomplexan parasites. Trends Parasitol. 33, 947-960.

32. Schaap, P., Schilde, C. (2018). Encystation: the most prevalent and underinvestigated differentiation pathway of eukaryotes. Microbiology, 164, 727-739.

33. Ali, V., Nozaki, T. (2006). Biochemical and functional characterization of phosphoserine aminotransferase from Entamoeba histolytica, which possesses both 
phosphorylated and non-phosphorylated serine metabolic pathways. Mol Biochem Parasitol 145, 71-83.

34. Kalhan, S. C., Hanson, R. W. (2012). Resurgence of serine: an often neglected but indispensable amino Acid. J Biol Chem 287, 19786-19791.

35. Deng, Y., Ran, W., Man, S., Li, X., Gao, H., Tang, W., Tachibana, H., Cheng, X. (2015). Artemether exhibits amoebicidal activity against Acanthamoeba castellanii through inhibition of the serine biosynthesis pathway. Antimicrob Agents Chemother 59, 4680-4688.

36. Perego, J., Bourbon, C., Chasson, L., Laprie, C., Spinelli, L., Camosseto, V., Gatti, E., Pierre, P. (2017). Guanabenz prevents d-galactosamine/lipopolysaccharide-induced liver damage and mortality. Front Immunol 8, 679.

37. Tsaytler, P., Harding, H. P., Ron, D., Bertolotti, A. (2011). Selective inhibition of a regulatory subunit of protein phosphatase 1 restores proteostasis. Science 332, 91-94.

38. Neuber, C., Uebeler, J., Schulze, T., Sotoud, H., El-Armouche, A., Eschenhagen, T. (2014). Guanabenz interferes with ER stress and exerts protective effects in cardiac myocytes. PloS one 9, e98893.

39. Anwar, A., Siddiqui, R., Hussain, M. A., Ahmed, D., Shah, M. R., Khan, N. A. (2018). Silver nanoparticle conjugation affects antiacanthamoebic activities of amphotericin B, nystatin, and fluconazole. Parasitology research, 117, 265-271.

40. Rajendran, K., Anwar, A., Khan, N. A., Siddiqui, R. (2017). Brain-eating amoebae: silver nanoparticle conjugation enhanced efficacy of anti-amoebic drugs against Naegleria fowleri. ACS Chem Neurosci 8, 2626-2630.

41. Sissons, J., Alsam, S., Stins, M., Rivas, A.O., Morales, J.L., Faull, J., Khan, N.A. (2006). Use of in vitro assays to determine effects of human serum on biological characteristics of Acanthamoeba castellanii. J Clin Microbiol 44, 2595-600. 
42. Lakhundi, S., Khan, N. A., and Siddiqui, R. (2014) Inefficacy of marketed contact lens disinfection solutions against keratitis-causing Acanthamoeba castellanii belonging to the T4 genotype. Exp Parasitol 141, 122-8

43. Sissons, J., Kim, K.S., Stins, M., Jayasekera, S., Alsam, S., Khan, N.A. (2005). Acanthamoeba castellanii induces host cell death via a phosphatidylinositol 3-kinasedependent mechanism. Infect Immun 73, 2704-2708.

44. Rajendran, K., Anwar, A., Khan, N. A., Shah, M. R., Siddiqui, R. (2019). transCinnamic acid conjugated gold nanoparticles as potent therapeutics against braineating amoeba Naegleria fowleri. ACS Chem Neurosci Article ASAP doi: 10.1021/acschemneuro.9b00111.

\section{Figure legends}

Figure 1. Guanabenz conjugated gold and silver nanoparticles were characterized by (a) UVVis spectroscopy (Thermo Scientific, Evolution 201) and (b and c) AFM (Agilent, 5500) respectively for GNB-AuNPs and GNB-AgNPs.

Figure 2. Antiamoebic activity of GNB and its conjugated gold and silver nanoparticles was evaluated against $A$. castellanii, as described in Methodology section. In brief, $A$. castellanii $\left(5 \times 10^{5} /\right.$ well / $500 \mu \mathrm{L}$ RPMI) trophozoites were treated with GNB alone and its nanoconjugates, for $24 \mathrm{~h}$, at $30{ }^{\circ} \mathrm{C}$. Number of viable $A$. castellanii were enumerated by trypan blue exclusion assay. The results are representative of three independent experiments carried out in duplicates as Mean \pm S.E.M. GNB conjugated gold and silver nanoparticles exhibited enhanced antiamoebic effects as compared to GNB alone $(* \mathrm{P}<0.05, * * \mathrm{P}<0.01$, while $* * * \mathrm{P}<0.001$; two-sample $\mathrm{t}$ test and two-tailed distribution). ${ }^{*}$ symbol represents the significance of GNB alone as compared to the untreated amoebae, whereas \# symbol 
represents the significance of 2.5 and $5 \mu \mathrm{M}$ concentrations of drug conjugated nanoparticles in comparison to the GNB alone.

Figure 3. Encystation of A. castellanii ( $5 \times 10^{5}$ / well / $500 \mu \mathrm{L}$ PBS+ Encystation Medium) was inhibited by incubation with GNB and its nanoconjugates at $30{ }^{\circ} \mathrm{C}$ for $72 \mathrm{~h}$. After incubation, $0.1 \%$ sodium dodecyl sulfate (SDS) was added to dissolve the remaining trophozoites and only amoebic cysts were counted, using a haemocytometer. The results are representative of three independent experiments carried out in duplicates as Mean \pm S.E.M ( $\mathrm{P}<0.05,{ }^{* *} \mathrm{P}<0.01$, while ${ }^{* * *} \mathrm{P}<0.001$; two-sample $\mathrm{t}$ test and two-tailed distribution). * symbol represents the significance of GNB alone as compared to the untreated amoebae, whereas \# symbol represents the significance of 2.5 and $5 \mu \mathrm{M}$ concentrations of drug conjugated nanoparticles in comparison to the bare nanoparticles and GNB alone.

Figure 4. Anticystic effects of GNB conjugated nanoparticles were observed against cysts of A. castellanii (1x105/ well /500 $\mu \mathrm{L}$ PYG). Briefly, amoebic cysts were incubated with GNB alone and its nanoconjugates at $30^{\circ} \mathrm{C}$ for $72 \mathrm{~h}$. After incubation, number of trophozoites were counted, using a haemocytometer. The results are representative of three independent experiments carried out in duplicates as Mean \pm S.E.M $(* \mathrm{P}<0.05, * * \mathrm{P}<0.01$, while $* * * \mathrm{P}<$ 0.001; two-sample $\mathrm{t}$ test and two-tailed distribution). * symbol represents the significance of GNB alone as compared to the untreated amoebae, whereas \# symbol represents the significance of 2.5 and $5 \mu \mathrm{M}$ concentrations of drug conjugated nanoparticles as compared to GNB and bare nanoparticles alone.

Figure 5. Pre-treatment of GNB alone and its conjugated gold and silver nanoparticles diminished A. castellanii mediated host cells cytotoxicity. Briefly, A. castellanii $\left(1 \times 10^{5} /\right.$ well / RPMI) trophozoites were treated with GNB alone and its nanoconjugates, for 2 hours, at 30 
${ }^{\circ} \mathrm{C}$. Then the pre-treated amoebae were incubated with $\mathrm{HaCaT}$ cells for $24 \mathrm{~h}$ at $37^{\circ} \mathrm{C}$ in a humidified 5\% $\mathrm{CO}_{2}$ incubator. Next day, the cytotoxicity was evaluated by using an LDH assay kit method (Roche). Negative control values for cytotoxicity assays were obtained by incubating HaCaT cells with RPMI-1640 alone were used as the negative controls, whereas $0.1 \%$ Triton X-100 treated cells were used as positive control depicting $100 \%$ cell death. The results are representative of three independent experiments carried out in duplicates as Mean \pm S.E.M $\left({ }^{*} \mathrm{P}<0.05, * * \mathrm{P}<0.01\right.$, while $* * * \mathrm{P}<0.001 ;$ two-sample $\mathrm{t}$ test and two-tailed distribution). * symbol represents the significance of GNB alone as compared to the untreated amoebae, whereas \# symbol represents the significance of 2.5 and $5 \mu \mathrm{M}$ concentrations of drug conjugated nanoparticles as compared to GNB and bare nanoparticles alone.

Figure 6. Antiamoebic activity of GNB alone and its nanoconjugates was evaluated against N. fowleri as explained in the Methods section. Briefly, $\left(5 \times 10^{5} /\right.$ well / $500 \mu \mathrm{L}$ HeLa + RPMI) N. fowleri trophozoites were treated with GNB alone and its nanoconjugates, for $24 \mathrm{~h}$, at 37 ${ }^{\circ} \mathrm{C}$ in a $5 \% \mathrm{CO}_{2}$ humidified incubator. Number of viable $N$. fowleri were counted by trypan blue exclusion using haemocytometer under an inverted microscope. The results are representative of three independent experiments carried out in duplicates as Mean \pm S.E.M. GNB conjugated gold and silver nanoparticles exhibited enhanced antiamoebic effects as compared to GNB alone $(* \mathrm{P}<0.05, * * \mathrm{P}<0.01$, while $* * * \mathrm{P}<0.001$; two-sample $\mathrm{t}$ test and two-tailed distribution). * symbol represents the significance of GNB alone as compared to the untreated amoebae, whereas \# symbol represents the significance of 2.5 and $5 \mu \mathrm{M}$ concentrations of drug conjugated nanoparticles as compared to GNB and bare nanoparticles alone. 
Figure 7. Anticystic effects of GNB alone and its nanoconjugates were evaluated against $N$. fowleri. Briefly, (1x105/ well / $500 \mu \mathrm{L}$ RPMI) N. fowleri cysts were incubated with different concentrations of GNB alone, GNB-Au and GNB-Ag for $72 \mathrm{~h}$, at $37{ }^{\circ} \mathrm{C}$ in a $5 \% \mathrm{CO}_{2}$ humidified incubator. After incubation time period, number of viable $N$. fowleri cysts were counted by trypan blue exclusion method using haemocytometer under an inverted microscope. The results are representative of three independent experiments carried out in duplicates as Mean \pm S.E.M. GNB conjugated gold and silver nanoparticles exhibited enhanced anticystic effects as compared to GNB alone (*or \#P $<0.05$; two-sample $t$ test and two-tailed distribution). * symbol represents the significance of GNB alone as compared to the untreated amoebae, whereas \# symbol represents the significance of 2.5 and $5 \mu \mathrm{M}$ concentrations of drug conjugated nanoparticles as compared to GNB and bare nanoparticles alone.

Figure 8. The cytotoxicity of GNB alone and its gold and silver conjugated nanoparticles was measured by LDH assay, as described in Methods section, against monolayers of (a) HeLa and (b) HaCaT cells. The results demonstrated that GNB and its nanoconjugates were non-cytotoxic against both the cell types. Next, cell-free supernatant was collected, and cytotoxicity was determined using an LDH assay kit (Roche). Negative control values for cytotoxicity assays were obtained by incubating HaCaT cells with RPMI-1640 only, whereas $0.1 \%$ Triton X-100 treated cells were used as positive control depicting $100 \%$ cell death. The results showed reduced host-cell cytotoxicity by the treatment of both GNB and its nanoconjugates. The results are representative of three independent experiments carried out in duplicates as Mean \pm S.E.M. 


\section{Page 27 of 34}

Figure 1.
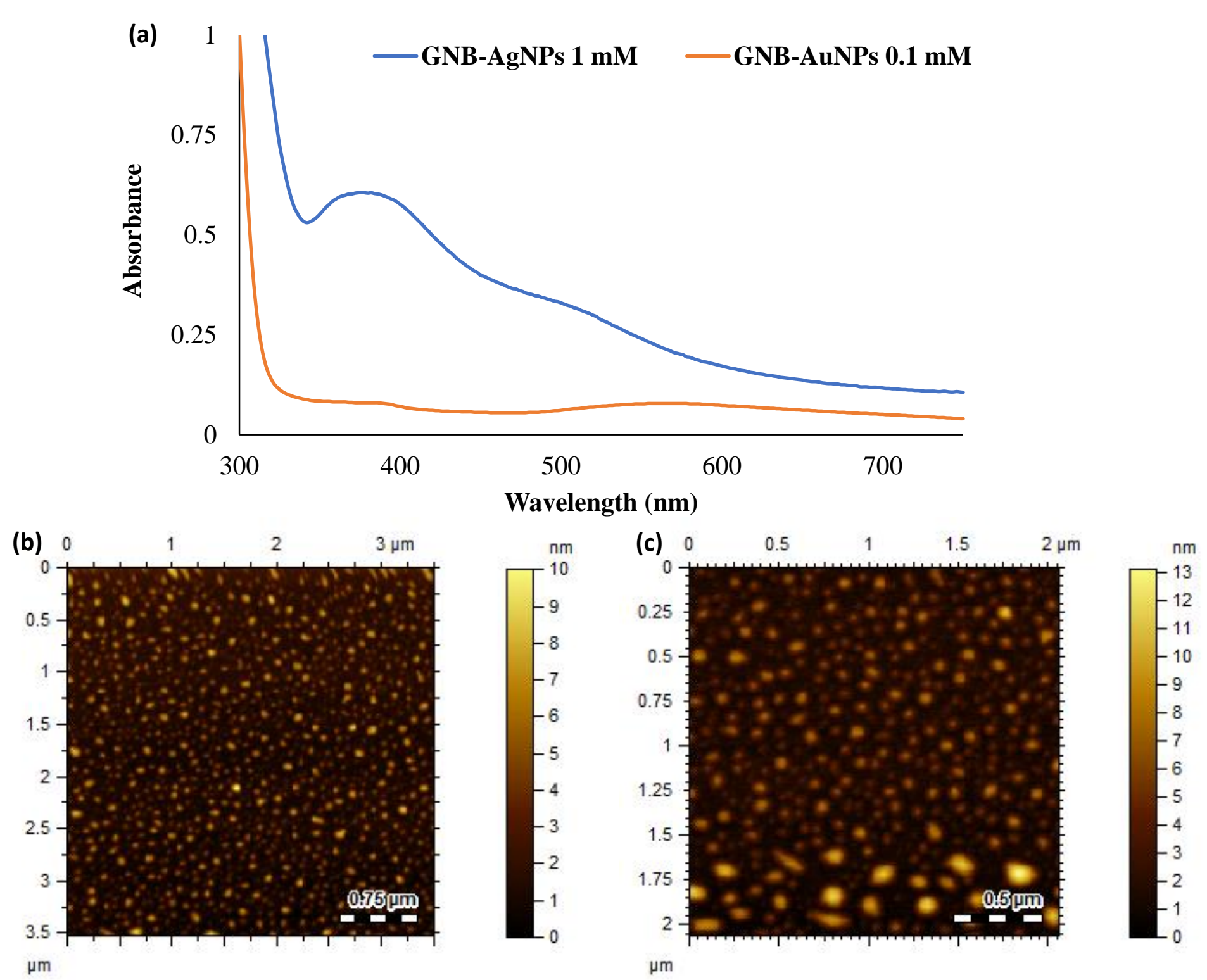


\section{Figure 2.}

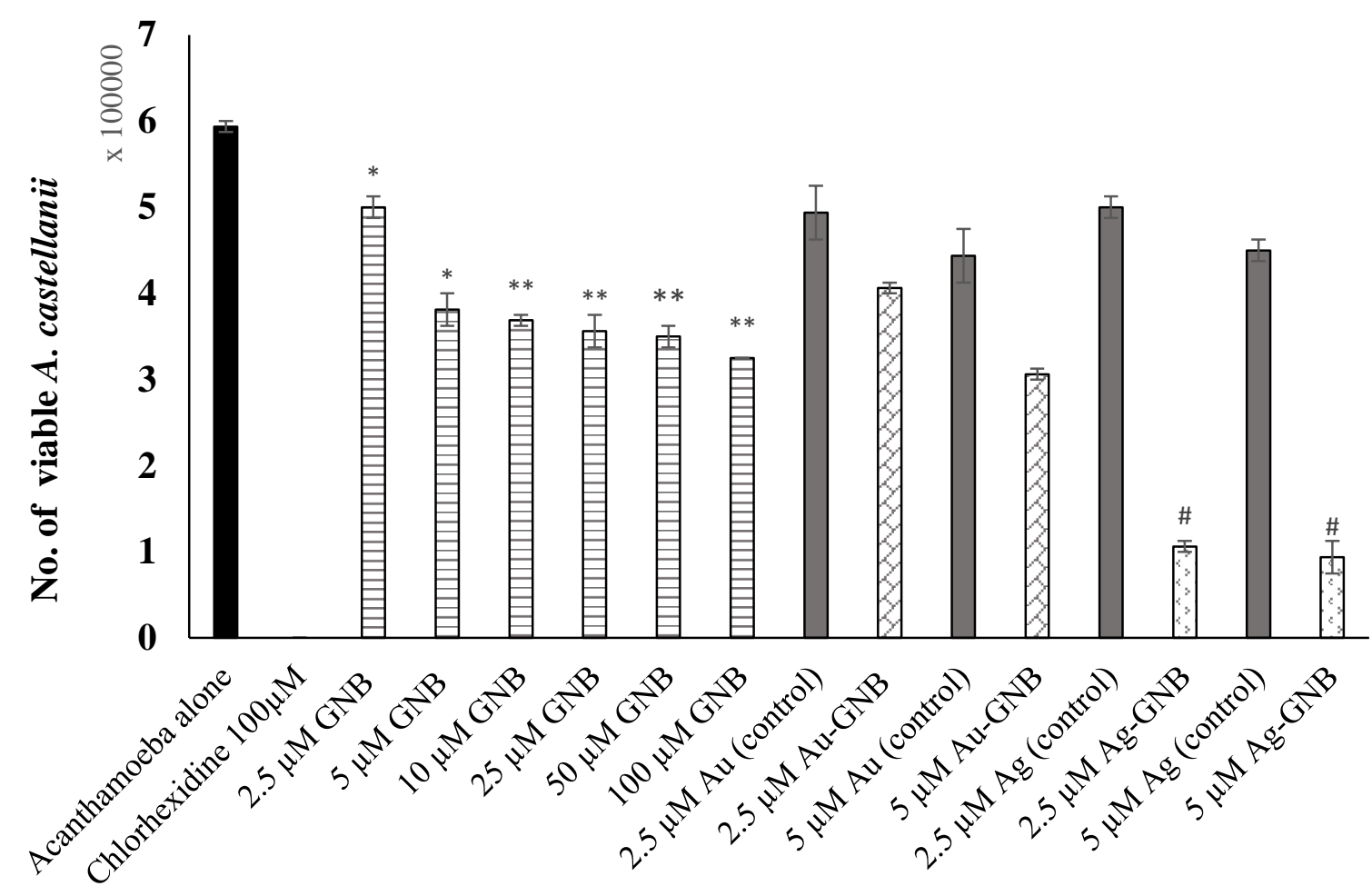




\section{Page 29 of 34
Figure 3.}

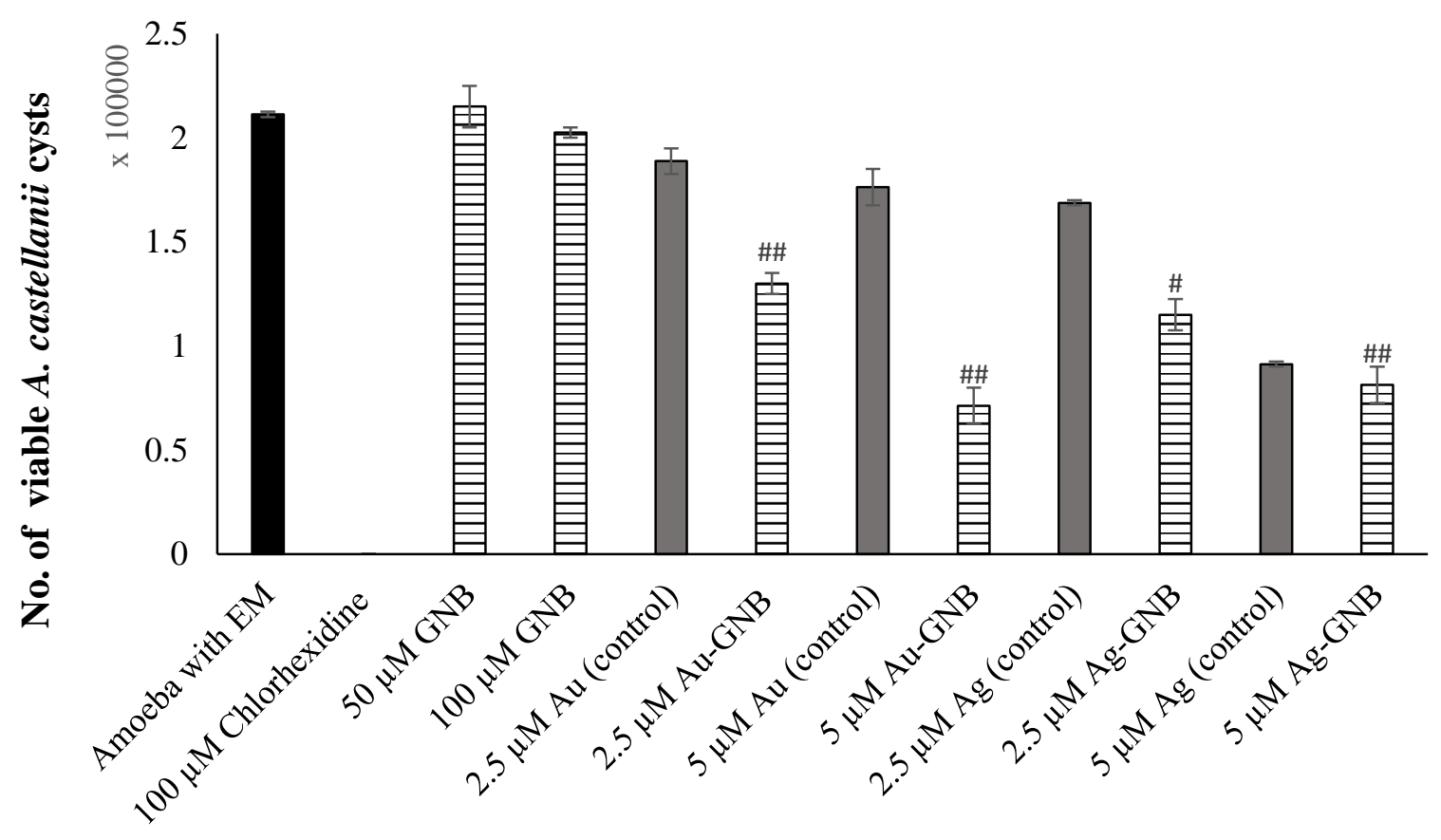




\section{Figure 4.}

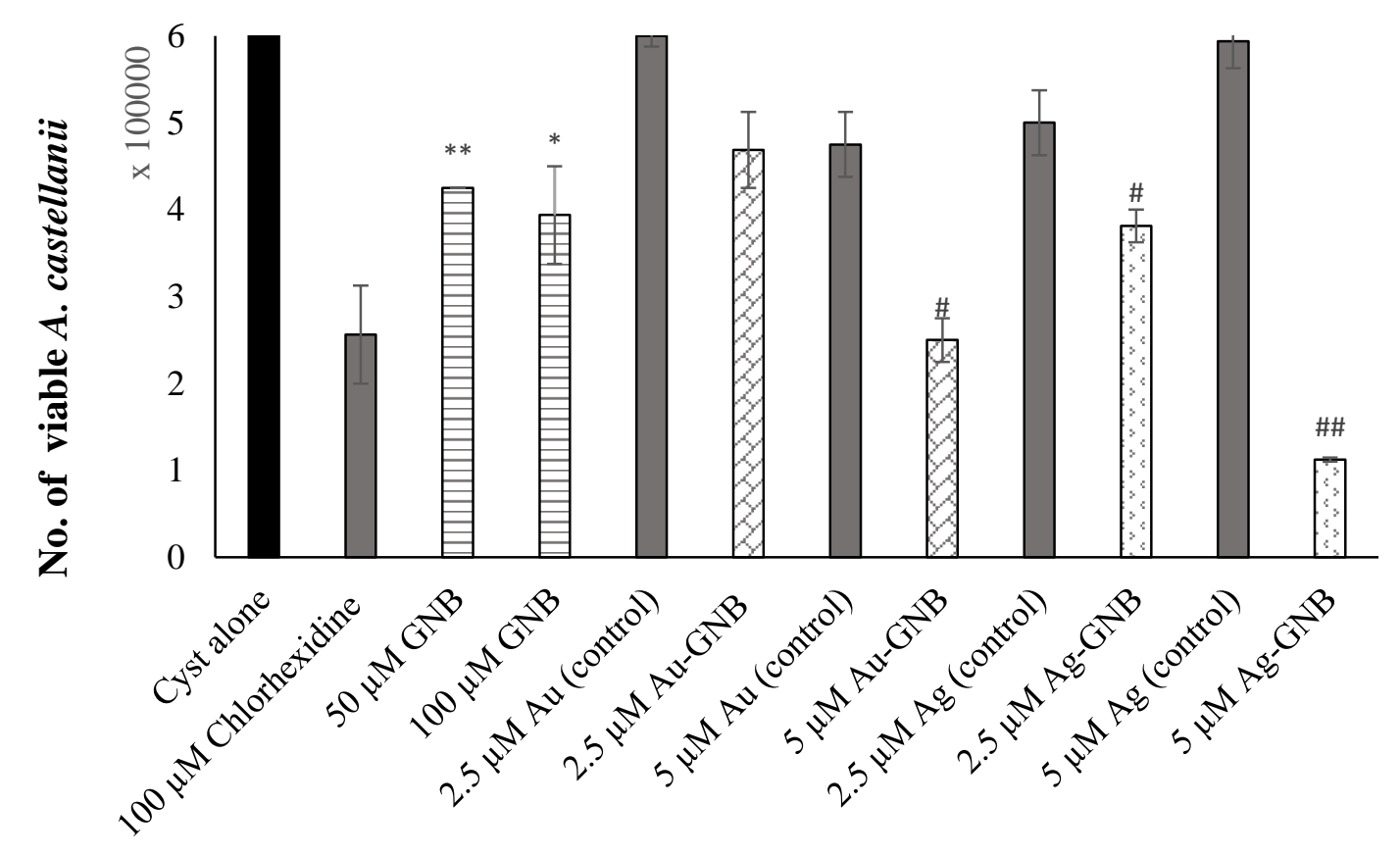




\section{Page 31 of 34}

Figure 5.

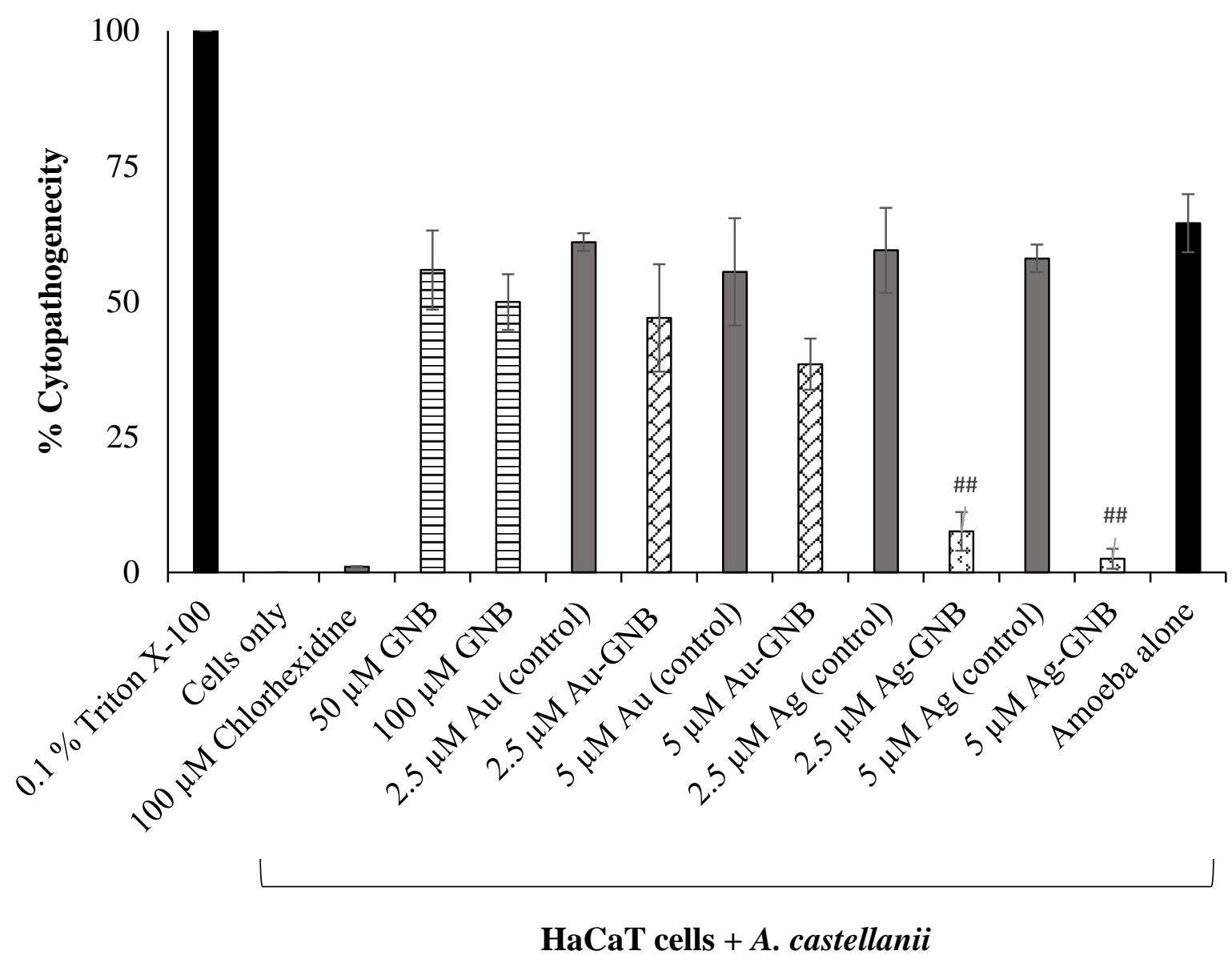




\section{Figure 6.}

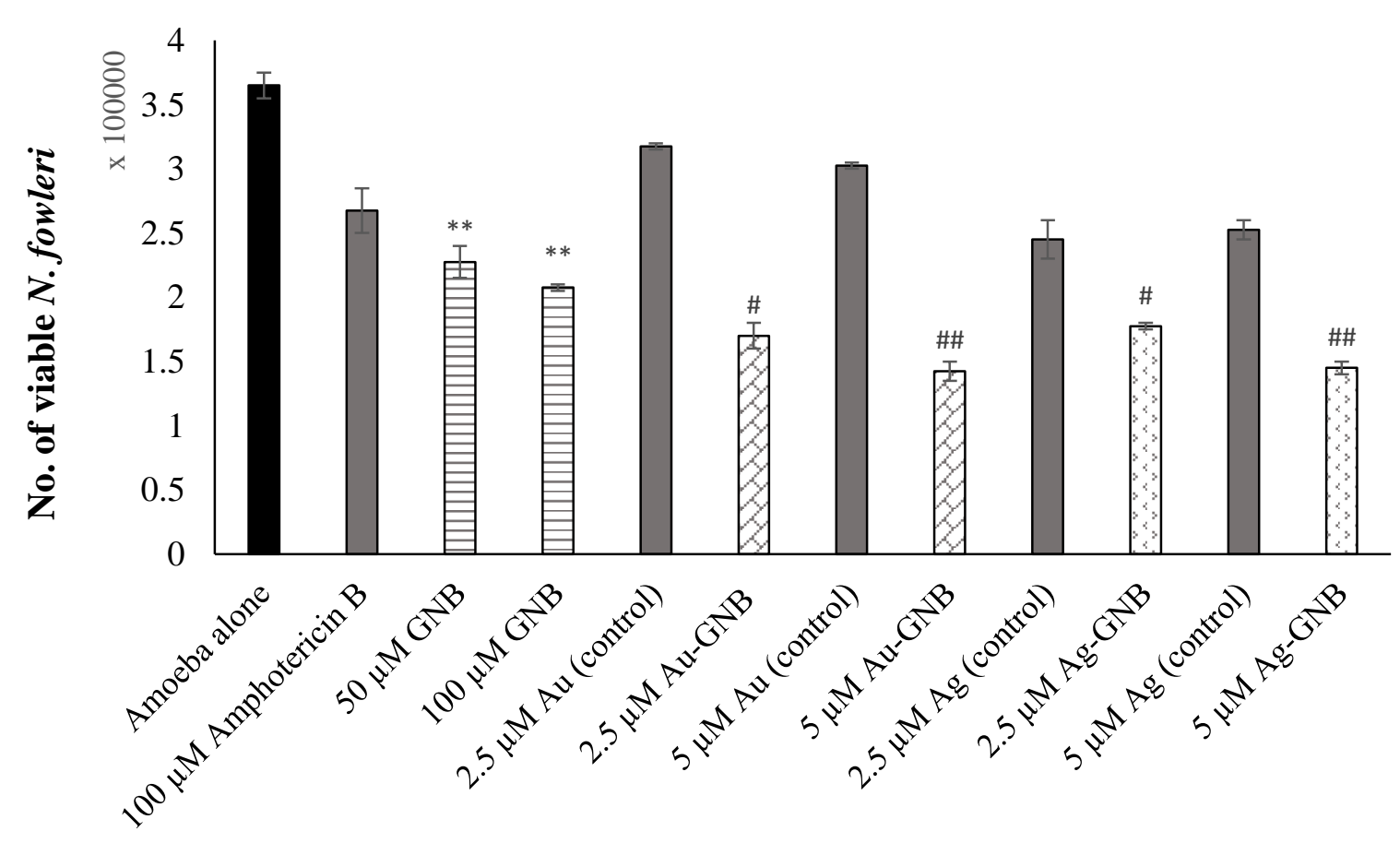


Figure 7.

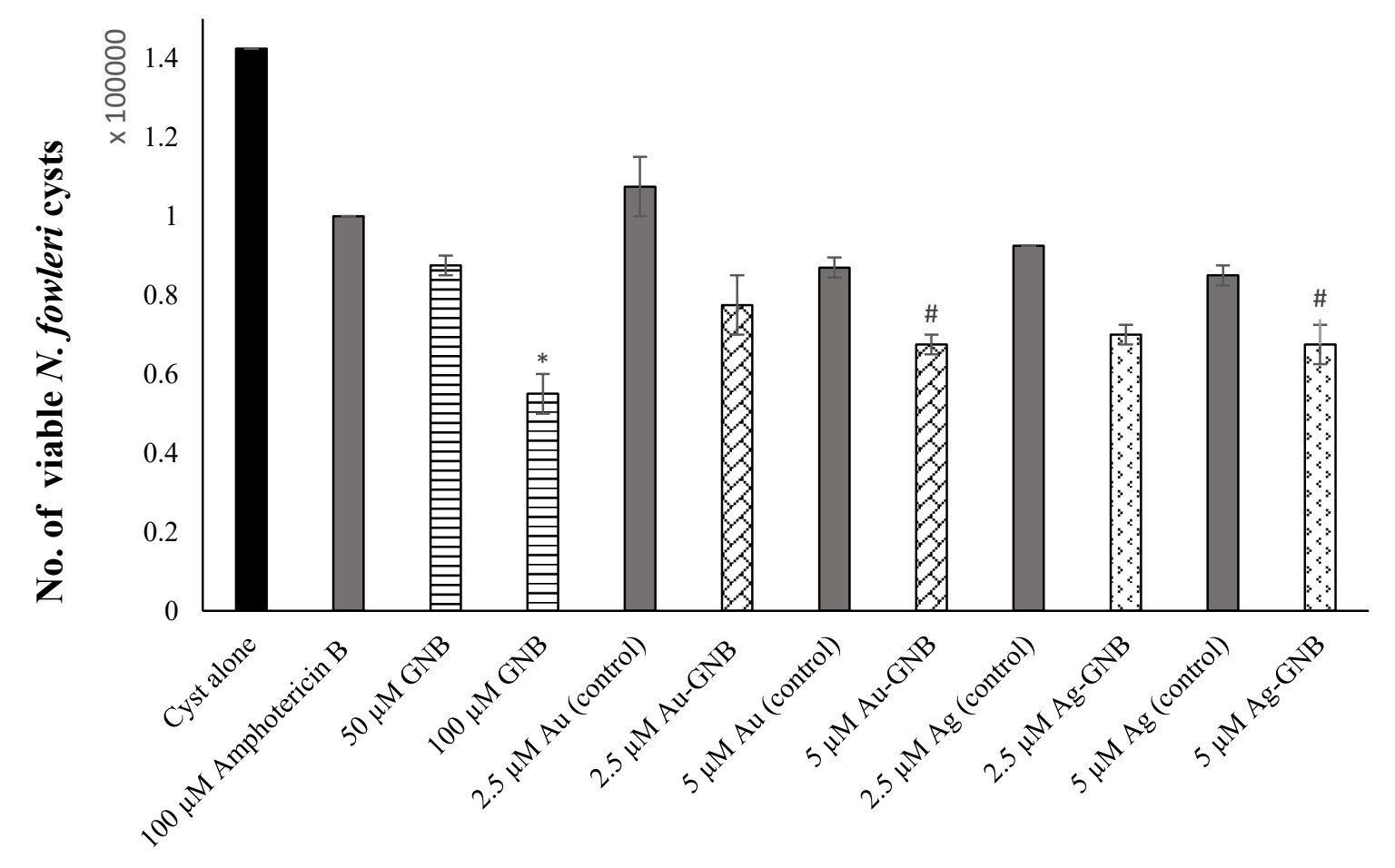




\section{Figure 8.}
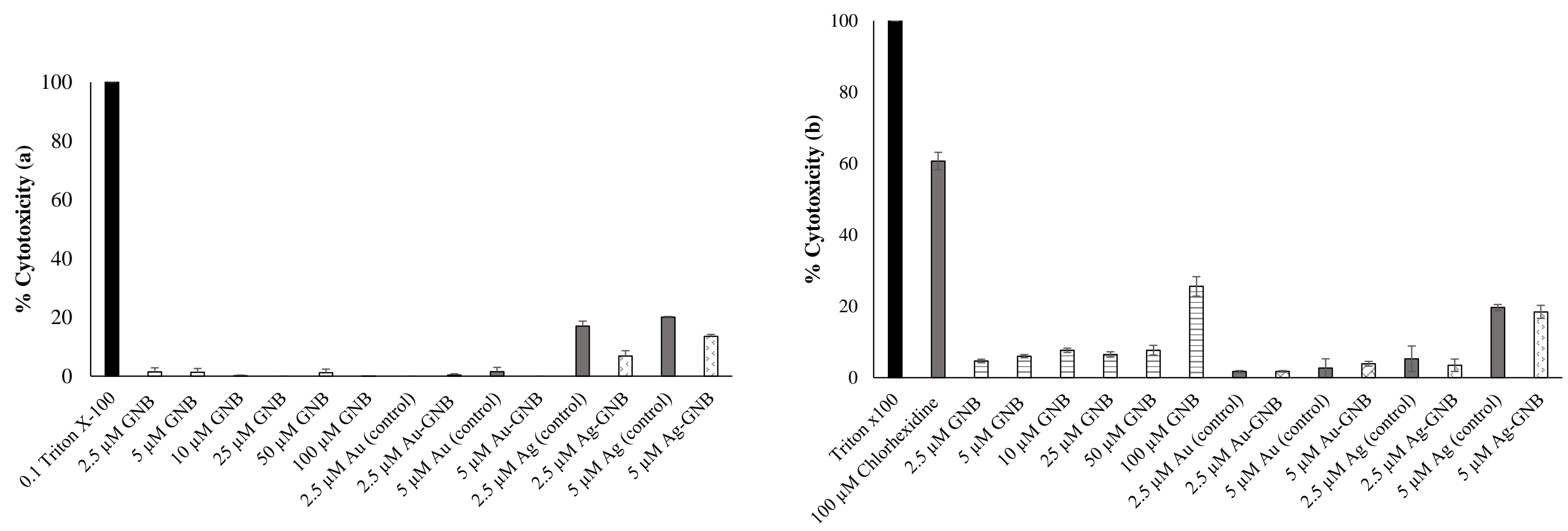